\title{
FIRST RESULTS OF ABSOLUTE MEASUREMENTS OF SOLAR FLUX AT THE IRKUTSK INCOHERENT SCATTER RADAR (IISR)
}

\section{A.G. Setov}

Institute of Solar-Terrestrial Physics SB RAS, Irkutsk,Russia,setov@iszf.irk.ru

\section{M.V. Globa}

Institute of Solar-Terrestrial Physics SB RAS, Irkutsk, Russia,globa@iszf.irk.ru

\section{A.V. Medvedev}

Institute of Solar-Terrestrial Physics SB RAS, Irkutsk,Russia,medvedev@iszf.irk.ru

\author{
R.V. Vasilyev \\ Institute of Solar-Terrestrial Physics SB RAS, \\ Irkutsk, Russia,roman_vasilyev@iszf.irk.ru
}

D.S. Kushnarev

Institute of Solar-Terrestrial Physics SB RAS, Irkutsk,Russia,ds_k@iszf.irk.ru

\begin{abstract}
The Irkutsk Incoherent Scatter Radar (IISR) allows us to carry out passive radio observations of the Sun and other powerful radio sources. We describe a method for absolute measurements of spectral flux density of solar radiation at IISR. The absolute measurements are meant to determine the flux density in physical units $\left[\mathrm{W} \cdot \mathrm{m}^{-2} \cdot \mathrm{Hz}^{-1}\right.$ ]. The IISR antenna is a horn with frequency beam steering, therefore radio sources can be observed at different frequencies. Also there is a polarization filter in the antenna aperture, which passes only single (horizontal) polarization. To acquire flux density absolute values, the IISR receiver is calibrated by the Cygnus-A radiation. Since the Sun's position in the IISR antenna pattern is determined by a frequency differing from the Cygnus-A observation frequency, we perform an additional calibration of the frequency re-
\end{abstract}

sponse in the 154-162 MHz operation frequency range, using the background sky noise. The solar disk size is comparable with the main beam width in the northsouth direction, hence the need to take into account the shape of the brightness distribution in the operation frequency range. The average flux density of the quiet-Sun radiation was $\sim 5 \mathrm{sfu}$ (solar flux units, $10^{-22} \mathrm{~W} \cdot \mathrm{m}^{-2} \cdot \mathrm{Hz}^{-1}$ ) at the $161 \mathrm{MHz}$ frequency.

Keywords: solar flux, absolute measurements, Irkutsk Incoherent Scatter Radar (IISR).

\section{INTRODUCTION}

The Irkutsk incoherent scatter radar (IISR) operates in a range 154-162 MHz and is used for ionospheric, satellite, and radio astronomical observations. The radar has been modernized - a digital reception system has been developed which can store received signal realizations in a complex representation [Potekhin et al., 2009]. This leverages post-processing of quadratures of received signals. The IISR antenna is a horn with dimensions of $246 \times 12 \mathrm{~m}$, divided by a partition into two half-horns with antenna pattern (AP) width of $0.5^{\circ} \times 20^{\circ}$. The antenna contains a polarizing filter, which passes only the horizontal polarization component of an incident wave. The radar has a frequency beam steering principle - the AP main lobe is tilted by $30^{\circ}$ from the vertical position to the south with frequency changed from 154 to $162 \mathrm{MHz}$. This facilitates passive observations of the Sun in summer as well as powerful radio sources (Cygnus-A, Cassiopeia, Crab Nebula) and background sky noise.

IISR has previously measured the received power in relative units [Vasilyev et al., 2013], but what is of scientific value for comparison with data from other instruments are absolute measurements of spectral flux density of solar radiation $S\left[\mathrm{~W} \mathrm{~m} \mathrm{mzz}^{-1}\right]$. It should be noted that by the absolute measurements are often meant measurements made without regular cali- bration by radiometers with known characteristics of antenna and receiver. In this paper, the absolute measurements refer to the determination of physical parameters after calibrating a receiver. The intensity and spatial distribution of solar radiation depend largely on frequency, but there are a small number of low-frequency instruments with large effective area. Moreover, during strong solar radio storms the radiation intensity in a low-frequency range can increase several hundred times. Absolute measurements of spectral flux density of solar radiation by various techniques have a long history [Baars, 2014]. However, the development of the IISR calibration algorithm required taking into account a number of peculiarities: frequency beam steering principle, great nonuniformity of the frequency response of the antenna system, and narrow bandwidth of feeder and receiver.

\section{METHOD FOR DETERMINING POWER FLUX DENSITY}

One of the most reliable and widely used calibration methods is the calibration by radiation from high-power radio sources (Cygnus-A, Cassiopeia-A, Taurus-A, Virgo-A) when the telescope beam size exceeds the size of the source [Baars, 2014]. An optimum reference source for IISR in terms of time and region of observation is 
Cygnus- $\mathrm{A}$. The Cygnus- $\mathrm{A}$ radiation spectrum has been much studied with various instruments, is employed for calibrating low-frequency telescopes, and can be approximated to arbitrary frequencies. We adopt the spectrum shape used for calibrating the LOFAR telescope operating in the 10-240 MHz range [Heald et al., 2015]

$$
\log S(f)=\log A_{0}+\sum_{i} A_{i} \log ^{i}(f / 150 \mathrm{M \Gamma ц}),
$$

where $A_{0}=10690 \mathrm{Jy}$ is the nominal power flux density of Cygnus-A at $150 \mathrm{MHz}, A_{1}=-0.67, A_{2}=-0.24$, and $A_{3}=0.021$ are spectral coefficients.

The spectral power flux density $S$ is expressed in terms of the radiation intensity $I$ :

$$
S=\int_{\text {Source }} I(\theta, \varphi) d \Omega .
$$

The receiver-input signal power $P_{\mathrm{r}}$ depends on AP and is defined by the expression

$$
P_{\mathrm{r}}=\frac{\Delta f G \lambda^{2}}{8 \pi} \int_{4 \pi} I(\theta, \varphi) F^{2}(\theta, \varphi) d \Omega,
$$

where $F^{2}(\theta, \varphi)$ is AP in the direction $(\theta, \varphi), G$ is the antenna gain. The IISR antenna passes only one polarization radiation component, expression (3) has, therefore, an additional term 0.5 . The shape of IISR AP $F^{2}(\theta, \varphi)$ has previously been determined and calibrated [Medvedev et al., 2002; Lebedev et al., 2006]. The gain $G$ depends on the beam slope and is computed for each operating frequency separately. To simplify expressions (2) and (3), we use the assumption about smallness of angular dimensions of the source compared to the main AP beam width or about lobe smallness compared to the source. These conditions hold for Cygnus-A, but the solar disk has angular dimensions of $\sim 0.5^{\circ}$ comparable with the IISR beam width; it is therefore necessary to set the shape of distribution of solar radiation intensity $I(\theta, \varphi)$. In a low-frequency range, the spatial distribution of solar radiation has a shape of an ellipse, and the distribution of the solar disk brightness decreases with distance from the center [Kundu et al., 1977]. For example, Leblanc, Le Squeren [1969] have shown that at a frequency of $169 \mathrm{MHz}$ during solar minimum the disk width is 32 arcmin in a north-south direction and 38 arcmin in an east-west direction. As a simple approximation of solar radiation intensity distribution we use a smoothed ellipse (Figure 1).

When propagating through the receiver, a signal is amplified and interfered with intrinsic receiver noise $b$, so the total power of the digitized signal takes the form

$$
P_{\mathrm{d}}=g\left(P_{\mathrm{r}}+b\right) \text {. }
$$

At present, passive observations with IISR are made in two operating modes: sky scanning and radio source tracking.

In the scanning mode, signals are received in turn at 11 frequencies covering the main frequency range. During the observation of the source, signals are received at a single frequency corresponding to the maximum of the model correlation coefficient between two half-horns of the radar [Vasilyev et al., 2016]. The shape of frequency response of receiver channels is largely determined by the antennafeeder path and analog part of the receiver, and is also limited by a digital filter with a bandwidth of $780 \mathrm{kHz}$.

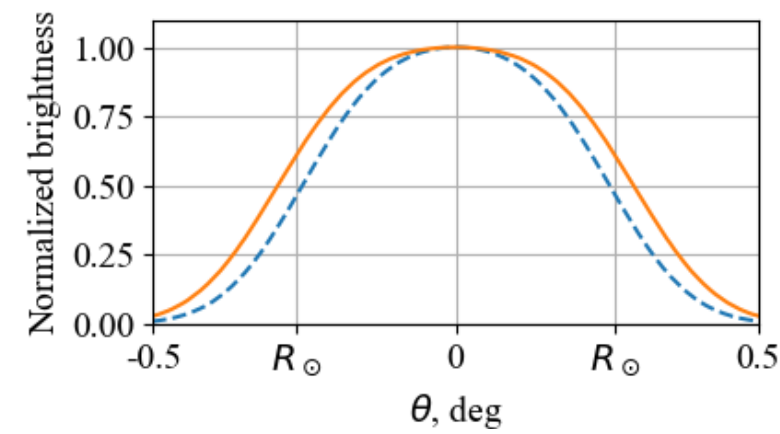

Figure 1. Model of radio brightness distribution over the solar disk. The solid line indicates the east-west direction; the dashed line, the north-south direction

The received time sequences are used to obtain the spectral power distribution, which corresponds to the spatial distribution, due to the frequency beam steering principle of IISR. Figure 2 shows a frequency-time distribution of the received uncalibrated power $P_{\mathrm{d}}$ for one day of observation during which two sources - the Sun (from 1 to 10 hrs) and Cygnus-A (from 15 to 20 hrs) were traced. In the scanning mode, 11 bandwidths are clearly identified; each of them defines the frequency response at a given central frequency. There is also an overall decrease in the power level (over $3 \mathrm{~dB}$ ) with the frequency varying from 162 to $154 \mathrm{MHz}$, which is significantly greater than the possible variations of the cosmic radio noise level in such a narrow frequency range.

The received power in the scanning mode includes cosmic radio noise and receiver noise. We can estimate the intrinsic noise levels $b$ and frequency distribution of the gain $g$ by fitting linear combination (4) to the model power at any operating frequency [Setov et al., 2017]. As the cosmic radio noise model we adopt the Global Sky Model (GSM), which allows us to obtain the sky noise distribution at an arbitrary frequency [de OliveiraCosta et al., 2008]. The level of the model cosmic noise for IISR varies during the day by a mean of $46 \%$ at a fixed frequency. The receiver noise level is twice as high as the mean sky noise level. The frequency variation in the sky noise level for a fixed time averages 34 $\%$. Figure 3 shows the estimated normalized frequency response averaged throughout the day. Red vertical lines indicate central receiving frequencies in the scanning mode (11 frequencies). The orange line marks the cubic spline interpolation of the gain at a central frequency.

Cosmic noise calibration allows us to consider the frequency-time dependence of the received noise power and determine the general shape of the frequency response of the IISR antenna. However, GSM sky maps are an approximation in wide frequency and spatial ranges and therefore contain errors. Moreover, the sky noise level, which serves as a reference signal during calibration, is comparable with the receiver noise level. To improve the calibration accuracy, we use a more stable and exact value of Cygnus-A radiation power, which exceeds the intrinsic noise levels seven times and the mean sky noise level fourteen times when the source is at a maximum of AP. Thus, the final calibration gain for solar radiation power is determined by Cygnus-A calibration and is multiplied by the difference in gain at frequencies of the Sun and Cygnus-A (Figure 3). 


\section{RESULTS OF MEASUREMENTS OF SOLAR RADIATION FLUX DENSITY}

After determining all calibration coefficients and evaluating AP integral (2), we can find spectral flux density of solar radiation (1) at the central frequency of observation bandwidth. Figure 4 illustrates July 05, 2017 solar radiation flux measurements. The flux density is expressed in solar flux units (sfu), $1 \mathrm{sfu}=10^{-22} \mathrm{~W} \mathrm{~m}$ ${ }^{2} \mathrm{~Hz}^{-1}$. In determining the flux density, we assume that the radiation is unpolarized; therefore, to obtain the full flux density, we double the received power. Since the AP position can be changed only in the north-south direction, the time of solar observation at a maximum of AP is limited to about one hour. Along the axis of the Figure is also the variation of the observation central frequency corresponding to solar observation during the day. The observed frequency range shifts from 162 $\mathrm{MHz}$ at a low position of the Sun to $160 \mathrm{MHz}$ during the summer solstice. The mean solar radiation flux density during the day of interest is $\sim 5 \mathrm{sfu}$, which is approximately half the total solar flux. At the Nancy telescope at a frequency of $169 \mathrm{MHz}$, the total flux of the quietSun radiation during low solar activity was 6 sfu [Leblanc, Le Squeren, 1969]. The solar radiation flux has high variability. For example, during the July 11, 2017 radio storm the solar radiation flux increased by a factor of hundreds compared to the flux from the quiet Sun (Figure 5). Estimating the error in the received powerflux density involves certain difficulties. An error in identifying the radiation flux is made by the error in evaluating calibration coefficients, the dispersion of the received power after averaging (1\%), inaccuracies in $\mathrm{AP}$, the error in cosmic noise model, temperature and humidity effects on characteristics of the antenna system (an error of $\sim 0.6$ arcmin per $1^{\circ} \mathrm{C}$ ).

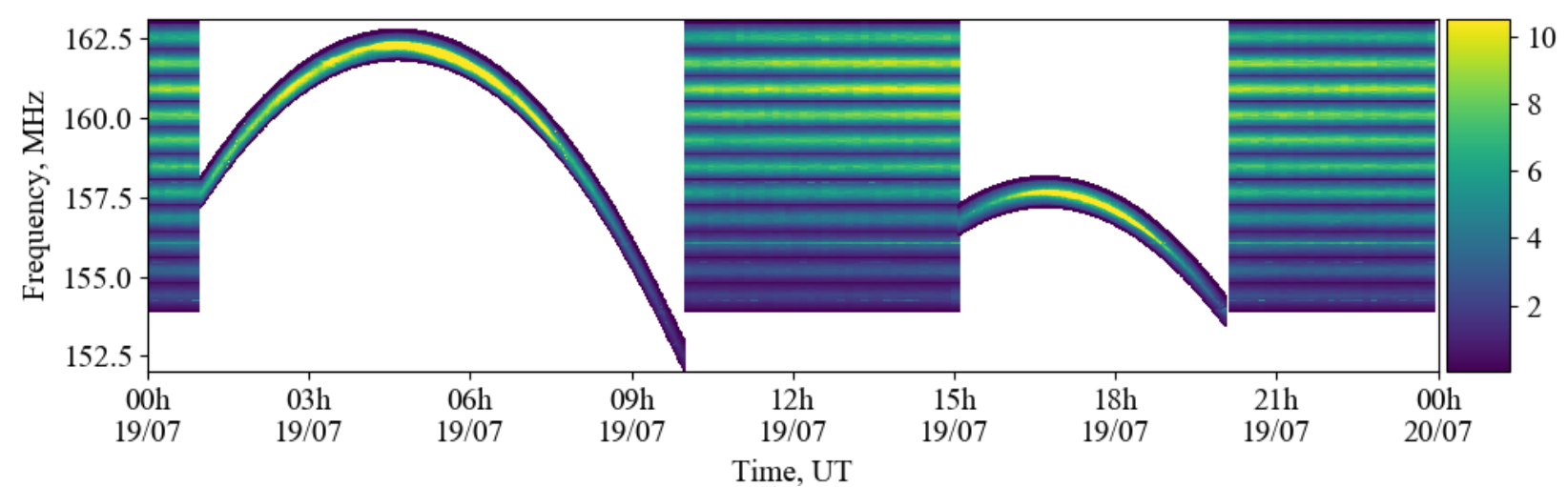

Figure 2. Frequency-time distribution of the received power $P_{\mathrm{d}}$ during the observation day (July 19, 2017). The color scheme is chosen in such a way as to place the emphasis on the background noise, so the power of the sources is beyond the boundaries of the scale (the power of the sources is much higher than the background noise level)

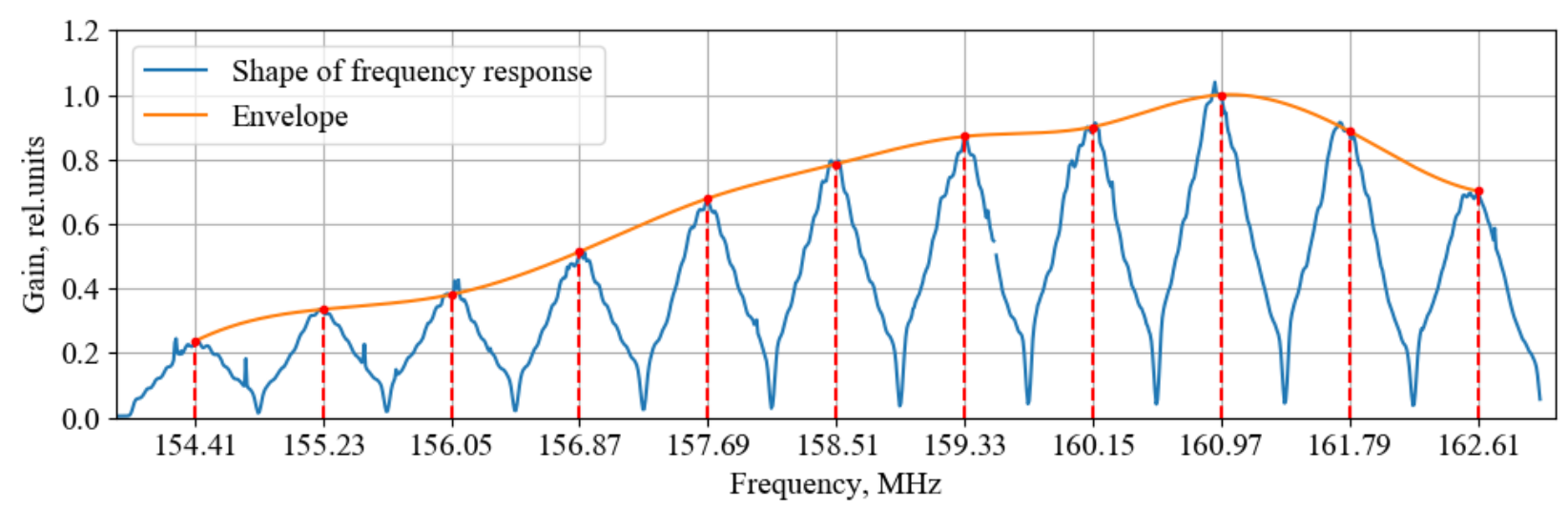

Figure 3. Estimated shape of frequency response of antenna and envelope at a central frequency 


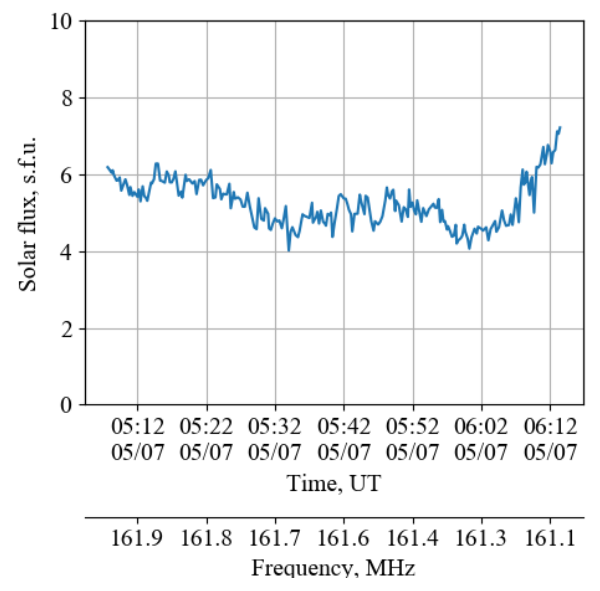

Figure 4. Spectral flux density of solar radiation on July 05, 2017

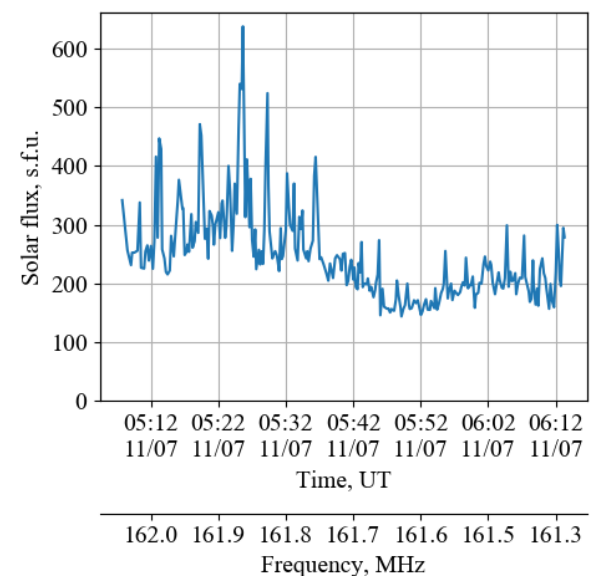

Figure 5. Spectral flux density of solar radiation on July11, 2017

A strong effect is also produced by the deviation of solar radio brightness distribution from the model ellipse (Figure 1). Therefore, further research is required to determine the accuracy of the estimated solar radiation power flux.

\section{|CONCLUSION}

The IISR antenna system and receiver have a nonlinear frequency response, which impedes absolute measurements of received power. The gain in the operating frequency range varies by $7 \mathrm{~dB}$ (Figure 3) and the frequency response in the receiver band has a varying shape depending on temperature of the antenna system. The proposed calibration method enables us to determine an exact gain at an arbitrary central frequency every day. For this purpose, we use the well-studied radio source Cygnus-A with known radiation flux, as well as GSM cosmic radio noise maps defining the shape of the frequency-time distribution of the background noise at IISR. The obtained spectral power flux density is $\sim 5 \mathrm{sfu}$ for the quiet Sun during low solar activity. We have also shown that IISR facilitates radiation flux measurements during strong solar radio storms (Figure 5).

The work was performed with budgetary funding of Basic Research program II.12 and under the project "Laying the Groundwork for National Heliogeophysical
Complex of the Russian Academy of Sciences”, unique number 0344-2018-0006. The results were obtained using the Unique Research Facility Irkutsk Incoherent Scatter Radar, http://ckp-rf.ru/usu/77733/.

\section{REFERENCES}

Baars J. History of flux-density calibration in radio astronomy. The Radio Science Bulletin. 2014, no. 348, pp. 47-66. DOI: 10.23919/URSIRSB.2014.7909943.

De Oliveira-Costa A., Tegmark M., Gaensler B.M., Jonas J., Landecker T.L, Reich P. A model of diffuse galactic radio emission from $10 \mathrm{MHz}$ to $100 \mathrm{GHz}$. Mon. Not. R. Astron. Soc. 2008, vol. 388. pp. 247-260. DOI: 10.1111/j.13652966.2008.13376.x.

Heald G.H., Pizzo R.F., Orru E., Breton R.P., et al. The LOFAR Multifrequency Snapshot Sky Survey (MSSS). I. Survey description and first results. Astron. Astrophys. 2015, vol. 582, pp. A123, 1-22. DOI: 10.1051/0004-6361/201425210.

Kundu M.R., Gergely T.E., Erickson W.C. Observations of the quiet Sun at meter and decameter wavelengths. Solar Physics. 1977, vol. 53, pp. 489-496. DOI: 10.1007/BF00160291.

Lebedev V.P., Medvedev A.V., Kushnarev D.S. Method to calibrate antenna pattern of Irkutsk IS radar. Trudy IX konferentsii molodykh uchenykh «Fizicheskie processy v kosmose i okolozemnoi srede» [Proc. of IX Young Scientists Conference "Physical Processes in Cosmos and Near Earth Space”]. Irkutsk, 2006, pp. 185-188. (In Russian).

Leblanc Y., Le Squeren A.M. Dimensions, temperature and electron density of the quiet corona. Their variations during the solar cycle. Astronomy \& Astrophysics. 1969, vol. 1, pp. 239-248.

Medvedev A.V., Zavorin A.V., Lebedev V.P., Lubyshev B.I., Nosov V.E. Incoherent scatter radar directional pattern using radio astronomical observations. Eighth International Symposium on Atmospheric and Ocean Optics: Atmospheric Physics. Proc. SPIE 4678. Irkutsk, 2002. DOI: 10.1117/12.458495.

Potekhin A.P., Medvedev A.V., Zavorin A.V., Kushnarev D.S., Lebedev V.P, Lepetaev V.V., Shpynev B.G. Recording and control digital systems of the Irkutsk Incoherent Scatter Radar. Geomagnetism and Aeronomy. 2009, vol. 49. no. 7, pp. 10111021. DOI: $10.1134 /$ S0016793209070299.

Setov A.G., Medvedev A.V., Lebedev V.P., Kushnarev D.S., Alsatkin S.S., Tashlykov V.P. Calibration methods for absolute measurements at the Irkutsk Incoherent Scatter Radar. 23rd International Symposium on Atmospheric and Ocean Optics: Atmospheric Physics. Proc. SPIE 10466. Irkutsk, 2017. DOI: $10.1117 / 12.2287328$.

Vasilyev R.V., Kushnarev D.S., Kashapova L.K., Lebedev V.P., Medvedev A.V., Nevedimov N.I., Ratovsky K.G. First results of radio observations of the Sun and powerful discrete sources at Irkutsk Radar. Astronomicheskii zhurnal [Astronomical journal], 2013, vol. 90, no. 11, pp. 948-958. (In Russian). DOI: $10.7868 /$ S0004629913110078

Vasilyev R.V., Globa M.V., Kushnarev D.S., Lebedev V.P., Medvedev A.V., Ratovsky K.G. Model of discrete cosmic radiosource signal for Irkutsk Incoherent Scatter Radar. XXV Vserossiiskaya otkrytaya konferentsiya «Rasprostranenie radiovoln» [XXV All-Russian Open Conference "Propagation of Radio Waves"]. Tomsk, 2016, vol. 3, pp. 122-125. (In Russian).

How to cite this article

Setov A.G., Globa M.V., Medvedev A.V. Vasilyev R.V., Kushnarev D.S. First results of absolute measurements of solar flux at the Irkutsk Incoherent Scatter Radar (IISR). Solar-Terrestrial Physics. 2018. vol. 4, iss. 3, pp. 24-27. DOI: $10.12737 /$ stp-43201804. 